\title{
Psychodrama an einer kinder- und jugendpsychiatrischen Versorgungs- und Universitätsklinik
}

\author{
Leonhard Thun-Hohenstein · Julia Trost-Schrems
}

Online publiziert: 19. Januar 2017

(C) Der/die Autor(en) 2017. Dieser Artikel ist eine Open-Access-Publikation.

Zusammenfassung Der Einsatz des Psychodramas an einer universitären Versorgungsklinik war anfangs mit Widerständen des Teams konfrontiert und konnte nach einer erfolgreichen Einstiegsgruppe als fixer Bestandteil des Behandlungskonzeptes etabliert werden. Eine der wichtigsten Erfahrungen ist, dass die Jugendlichen gut profitieren, allerdings oft bereits die sanften und einfachen Techniken wie Aufwärmübungen ausreichen, um hilfreich zu sein.

Schlüsselwörter Psychodrama - Gruppentherapie · Psychisch kranke Jugendliche · Stationärer Kontext

\section{Use of psychodramatic group therapy for inpatients in a child and adolescent psychiatric department}

\begin{abstract}
The introduction of psychodrama in a university child and adolescent clinic met with resistance from the therapeutic team. After a successful probationary period psychodrama was established as a fixture in the treatment programme for eating disordered and other mentally ill young people. An important learning was that these young patients benefit from simple and gentle techniques, such as warmup exercises, which are sufficient and useful.
\end{abstract}

Keywords Psychodrama · Group therapy · Mentally ill adolescents · Inpatient treatment

Prim. Univ.Prof. Dr. L. Thun-Hohenstein $(\varangle) \cdot$ J. Trost-Schrems

Univ.Klinik f. Kinder + Jugendpsychiatrie, Universitätsklinikum Salzburg/CDK,

Ignaz-Harrerstr.79, 5020 Salzburg, Österreich

E-Mail: 1.thun-hohenstein@salk.at 


\section{Einleitung und Hintergrund}

Psychodramatische Gruppentherapien mit Kindern und Jugendlichen werden häufig im ambulanten Setting angeboten und verwirklicht (Bindernagel et al. 2011, Altendorfer-Kling et al. 2011). Das ist einerseits mit der guten Wirksamkeit im ambulanten Setting begründet und andererseits mit einem geringen Anteil an klinisch tätigen Psychodrama-Therapeuten zu erklären. In den letzten Jahren scheint diese Zahl zugenommen zu haben, so auch an unserer Klinik.

Die Universitätsklinik für Kinder- und Jugendpsychiatrie ist eine Versorgungsklinik mit einem Einzugsgebiet von 500.000 Einwohnern. Sie verfügt über 20 stationäre und 10 tagesklinische Betten. Die grundlegende Ausrichtung fußt auf systemischen Grundsätzen und lässt sich in 4 wesentlichen Paradigmen beschreiben: Ressourcenorientierung, Perspektivenwechsel, Ziel- und Lösungsorientierung und als Basis Strukturen, die Mitarbeit ermöglichen. Zentrales Ziel dieser Strukturen ist die größtmögliche Partizipation der Patientinnen und ihrer Angehörigen (Thun-Hohenstein 2014). In Abb. 1 wird diese Gesprächsstruktur übersichtlich dargestellt. Im Rahmen dieser Gespräche werden die Ziele für die Behandlung festgelegt, die Ressourcen mobilisiert und vereinbart, wer, was, zu welchem Ziel beitragen kann. Gleichzeitig werden jeweils der Fortschritt oder Schwierigkeiten des Behandlungsprozesses evaluiert und besprochen und entsprechende Anpassungen der Ziele oder des Behandlungsprozesses vorgenommen. In diesem Rahmen wird auch die Indikation zur Psychodramagruppe gestellt.

Das Psychodrama wird bei uns in mehrfacher Weise eingesetzt: in der Einzeltherapie (Monodrama), in der stationären Gruppentherapie (allgemein supportive Gruppe und Essstörungsgruppe) und als therapeutische Gruppe während des Sommerpro-

Abb. 1 Gesprächsstruktur als Partizipations- und Entscheidungsgremium für den therapeutischen Prozess

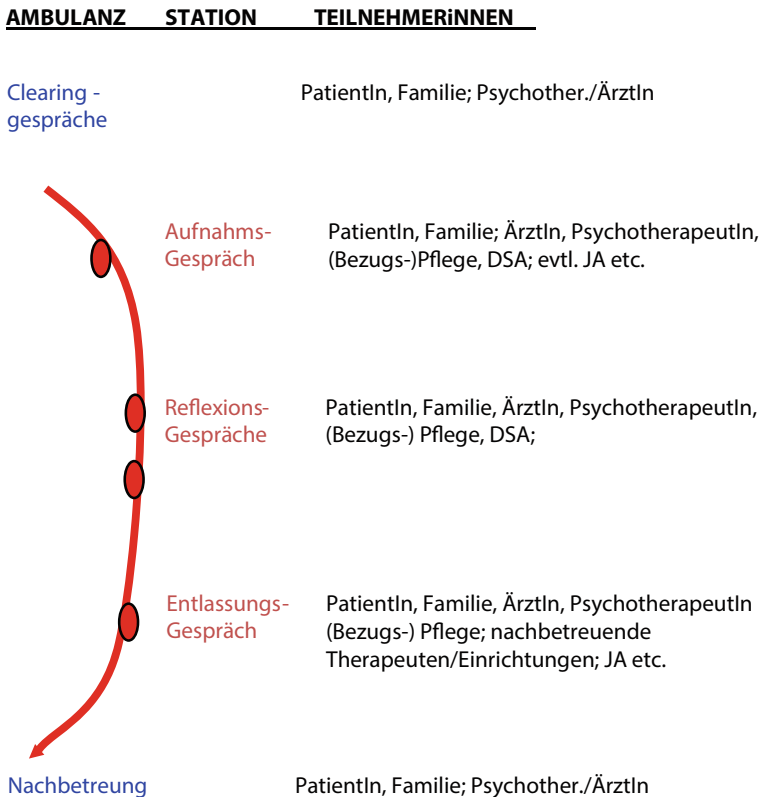


jektes für Patientinnen mit einer Essstörung. Das Sommerprojekt umfasst einen sechswöchigen Aufenthalt an der Tagesklinik der Universitätsklinik für Kinder- und Jugendpsychiatrie. Die Patientinnen waren zuvor noch nicht in teil- oder vollstationärer Behandlung, sie erfüllen die Kriterien einer Essstörung, ihr Gewicht liegt jedoch über der 3. BMI-Perzentile.

Unsere Patientinnen sind zwischen sechs und 18 Jahre alt und leiden an mindestens einer psychiatrischen, mehr als die Hälfte aber mindestens an zwei psychiatrischen Erkrankungen. Sie sind meist massiven psychosozialen Belastungen ausgesetzt und vielfach traumatisiert. Viele erhalten Medikamente, alle haben Psychotherapie und mindestens drei weitere therapeutische Angebote.

\section{Indikationen zum Psychodrama}

In die allgemein-supportive Gruppe werden PatientInnen ab einem Alter von 14 Jahren von Station und Tagesklinik gemeinsam aufgenommen. Die Teilnahme wird nach ausführlicher Diskussion im therapeutischen Team - im Reflexionsgespräch festgelegt. Ziel dieser Gruppe ist es die alltäglichen Probleme der Jugendlichen zu begleiten, die soziale Kompetenz zu stärken, Konflikte zu bearbeiten und den sozialen Raum als Therapeutikum zu nutzen und zu unterstützen. Die Gruppe wird halboffen geführt, sodass - nach Rücksprache mit den Therapeutinnen - jederzeit ein neues Mitglied aufgenommen werden kann. Themen sind zum Beispiel Selbstwertund Ich-Stärkung, Konflikte mit MitschülerInnen/LehrerInnen/Eltern, Entspannung, Umgang mit Stress, Erprobung sozialer Fertigkeiten.

Die stationäre Essstörungsgruppe ist ebenfalls als supportive Gruppe angelegt und findet statt, wenn zu viele negative Interaktionen und gegenseitige Belastung durch die Essstörung entstehen. Themen sind essstörungsfreie Räume, Verhinderung des gegenseitigen Negativ-Wettbewerbs (z. B. bei Essverhalten, Selbstverletzung o. ä.), Beziehungsgestaltung abseits der Essstörung, Interaktion mit dem Behandlungsteam, Konflikte.

Die Essstörungsgruppe im Sommerprojekt der Tagesklinik wird als zentraler Bestandteil der Therapie angeboten und ist eine klassische Psychodramagruppe, modifiziert für Jugendliche nach dem Modell von Alfons Aichinger (Aichinger und Holl 1997). Themen sind Selbstwert- und Ich-Stärkung, Körperbild, Essen und Essenssituationen sowie familiäre Konflikte.

Das Psychodrama in der Einzeltherapie wird als Teil einer umfassenden kinderund jugendpsychiatrischen Behandlung angewandt und entsprechend der Thematik und den Ressourcen der PatientInnen eingesetzt.

\section{Verwendete Techniken und Therapieablauf in der Gruppe}

a) Aufwärmrunde:

- mit Befindlichkeitsrunde: Fischkarten mit verschiedenen Gesichtern, Stimmung als Wetterbericht, Stimmungsfarbe, etc., 
- Aufwärmen mit Gegenständen,

- Zeichnen/Knetmasse/Handpuppen etc.

b) Erarbeiten von Gruppenregeln (soziale Regeln, Verschwiegenheit etc.), die alle unterzeichnen.

c) Spielphase:

- „Psychodrama-Stille-Post“ = pantomimisch;

- „Rundumgeschichte“ (,,es war einmal ein ...“, jeder ergänzt einen Satz, danach Spielen der Geschichte),

- ,eine Person/Tier in das ich heute schlüpfen möchte“,

- Märchen (jeder nennt Lieblingsmärchen, Abstimmung welches gespielt werden soll, freie Rollenwahl),

- Schiffsreise: freie Rollenwahl (Koch, Tourist, Kellner, Kapitän ...),

- „Speed-Dating“ mit selbst gewählten Rollen,

- spontanes Spiel mit selbstgewählten Rollen (Treffen im Park, Gasthaus, Cafe ...),

- „Sichere Insel“ (jeder sucht sich Platz im Raum und baut für sich sichere Insel/Ort),

- „Dolmetscherspiel“",

- ProtagonistInnenspiele: selten, meist nach Vorbesprechung im spezifischen Einzelsetting,

- „Begegnung und Begrüßung als ....“ (Schüler, Pfarrer, Nonne, Verkäufer am Markt, Pensionisten, .....).

d) Rollenfeedback und Sharing.

\section{Leitertechniken}

Das Spiegeln ist die meist eingesetzte Technik, da sie eine sehr behutsame Form der Rückmeldung darstellt und in erster Linie dem positiven Ich-Erleben dient und zur Selbstreflexion anregt. Teilweise erfolgt das Spiegeln auch wie beim KinderPsychodrama durch ein mitspielendes erwachsenes Hilfs-Ich (Aichinger und Holl 1997). Doppeln wird ausschließlich im Sinne des einfühlenden Doppelns angewandt mit dem Ziel den Jugendlichen zu ermöglichen, wahrzunehmen was in ihnen vorgeht, Worte dafür zu finden und dies zu teilen. Begeistern lassen sich Jugendliche oft für das Rollenspiel, wobei der Rollenwechsel weniger beliebt ist, möglicherweise wegen der durch die Grunderkrankung verunsicherten Persönlichkeit. Dennoch ist der Rollenwechsel eine wesentliche Technik bei der Bearbeitung von Beziehungskonstellationen und Konflikten.

Bei den häufig mehrfachtraumatisierten Jugendlichen ist die Schaffung einer sicheren Struktur und Gruppenkultur zentral. Das gegenseitige Beobachten und Austauschen über den „,sicheren Ort“, das Erleben des Respekts der anderen vor diesem, das Einüben des Einforderns der Grenzeinhaltung sind ganz wesentliche Bestandteile der Therapie in diesem Setting. Diese Erfahrungen haben ganz wesentlichen Einfluss auf das Stationsleben wie auch das Leben draußen, setzen allerdings das Vorhandensein oder zumindest ein Verständnis dieses Konzeptes in den erwähnten Kontexten voraus. 


\section{Erfahrungen}

Die Leitung dieser Gruppen erfordert einerseits eine sehr genaue Kenntnis der jeweiligen Krankheitsbilder, ihrer Symptome und vor allem verlangt es ein großes Wissen darüber, wie akut auftretende psychopathologische Symptome zu verstehen und einzuordnen sind. Es ist auch in der Vorbereitung zu überlegen, welche Phänomene (z. B. Dissoziation, Isolation, Halluzinationen, Suizidalität, Regression/Aggression, Selbstverletzungstendenzen etc.) zu erwarten sein könnten und wie mit diesen umzugehen sein wird. Der/die Leiterin und ihre/seine Co-Leitung muss jederzeit in der Lage sein, für die Gruppe und ihre Mitglieder die nötige Struktur zu halten und für körperliche und seelische Sicherheit zu sorgen.

Zentral dabei ist die Fähigkeit das emotionale und situative Containing anzubieten. Darunter verstehen wir nach Bion die Funktion des Aufnehmens und ,Verwandelns" unerträglicher seelischer Inhalte in erträgliche Inhalte. Die Gruppe selbst ist Teil dieses Containing durch das Mitspielen, Mitfühlen, Reflektieren und Sharing, kann das aber - gerade bei psychisch Kranken - nur indem die Leitung die entsprechenden Funktionen wahrnimmt.

Eine andere Funktion der Leitung ist die Anleitung der Gruppe zur Mentalisierung. Als Mentalisierung wird nach Fonagy (Allen et al. 2011) die Entwicklung eines Verständnisses für mentale Zustände sowie deren Inhalte wie Bedürfnisse, Wünsche, Gefühle und Gedanken verstanden. Es geht auch um das Explizit-Machen des Unbewussten, das sich im Spiel ausdrückt und das ins Bewusstsein-Holen des eigenen Narrativs. Gerade für diese Fragestellung bietet das Psychodrama ausreichend Möglichkeiten aus dem Erleben im Spiel, dem Rollenfeedback und dem Sharing ein Übungsfeld für das Mentalisieren. Die Technik des Mentalisierens hat mittlerweile als eigene Therapieform Eingang in die Kliniken gefunden (Jacobsen 2014) und scheint gerade bei depressiven, anorektischen Patientinnen oder solchen mit Persönlichkeitsstörungen ausgesprochen hilfreich zu sein.

Die Arbeit mit psychisch kranken Jugendlichen erfordert eine deutlich niedrigere Erwartungshaltung des Therapeuten an die Umsetzung des erworbenen Wissens und der Technik. Es sind gerade die „kleinen“ und „sanften“ Techniken, die bei den Jugendlichen häufig den meisten Effekt haben. Das Aufwärmen mit seinem bunten Strauß an Möglichkeiten füllt oft in der Erlebnistiefe und Aufarbeitung eine ganze Therapieeinheit ohne dass es je zu einem Protagonisten- oder Gruppenspiel kommt. Selbstgestaltete Rollenspiele sind ein bei den Jugendlichen äußerst beliebtes Mittel um ihre Welt in spielerischer Weise zu inszenieren und zu teilen.

\section{Integration in den Klinikalltag}

Anfangs gab es deutliche Widerstände des Behandlungsteams noch eine zusätzliche Therapie einzuführen. Ein besonders wirksames Argument dagegen war die Prämisse, dass es nicht gleichzeitig zwei oder mehr psychotherapeutische Interventionen am selben Patienten geben darf. Außerdem würde es die Möglichkeiten der Jugendlichen nach Rückzug einschränken, sie möglicherweise in schwierige Stimmungen versetzen, die dann das Betreuungsteam aufzufangen habe. Es wurde daher also 
zuerst die supportive Gruppe für Jugendliche mit Essstörungen angeboten und damit konnte auch die Wirksamkeit und Sinnhaftigkeit dieser Methode nachgewiesen werden. Infolge wurden dann die übrigen Angebote eingeführt und sind mittlerweile hoch akzeptierte Teile unseres therapeutischen Angebots.

Dabei ist wichtig festzuhalten, dass die Erfahrungen der Psychodrama-therapeutInnen in die Gesamtteambesprechungen einfließen. Dies ist wichtig um die oft von anderen therapeutischen Settings abweichenden Erfahrungen zu kommunizieren und so ein breiteres Bild der Jugendlichen und ihrer Ressourcen und Fähigkeiten wie auch ihrer Belastbarkeit zu entwickeln. Für die Akzeptanz des Psychodramas ist es aber ebenso wichtig einen Ausdruck für die Tätigkeit und die Qualität dieser Methode zu finden umso über die erlebte/erlebbare Sinnhaftigkeit dieser Methode die Akzeptanz und Bedeutung zu erhöhen.

\section{Zusammenfassung}

Psychodrama kann ausgezeichnet in den Alltag einer Versorgungsklinik integriert werden und in verschiedenster Form und Intention eingesetzt werden. Unsere Angebote umfassen sogenannte „supportive“ Gruppen für die stationär aufgenommen Patientinnen oder in spezifischen schwierigen Situationen, z. B. bei EssstörungspatientInnen. Voraussetzungen für die Durchführung sind neben den klassischen Bedingungen (Indikation, Freiwilligkeit, Raum etc.) die Akzeptanz im gesamten therapeutischen Team sowie das genaue Verständnis der Psychopathologie und ihrer möglichen (positiven wie negativen) Auswirkungen auf das Gruppengeschehen. Als letzter Punkt ist die Reintegration der Gruppenerfahrungen in das Gesamtbehandlungskonzept von zentraler Bedeutung.

Open access funding provided by Paracelsus Medical University.

Open Access Dieser Artikel wird unter der Creative Commons Namensnennung 4.0 International Lizenz (http://creativecommons.org/licenses/by/4.0/deed.de) veröffentlicht, welche die Nutzung, Vervielfältigung, Bearbeitung, Verbreitung und Wiedergabe in jeglichem Medium und Format erlaubt, sofern Sie den/die ursprünglichen Autor(en) und die Quelle ordnungsgemäß nennen, einen Link zur Creative Commons Lizenz beifügen und angeben, ob Änderungen vorgenommen wurden.

\section{Literatur}

Aichinger, A., \& Holl, W. (1997). Psychodrama-Gruppentherapie mit Kindern. Mainz: Mathias Grünewald Verlag.

Allen, J.G., Fonagy, P., \& Batemean, A.W. (2011). Mentalisieren in der psychotherapeutischen Praxis (S. 49). Stuttgart: J.G.Cotta'sche Buchahndlung \& Nachfolger.

Altendorfer-Kling, U., Herzog-Schuster, B., \& Thun-Hohenstein, L. (2011). Siegen, wenn die Chanecn schlecht stehen! - Psychodramagruppe für Kinder mit psychosozialen Schwierigekeiten und Kinder von psychisch kranken Eltern. Z Psychodrama und Soziometrie, 3, 125-140. Sonderheft.

Bindernagel, D., Scherrer, A., Winzeler, A. L., \& von Wyl, A. (2011). Psychodrama Gruppentherapie mit Kindern - Anregungen für eine praxisorientierte Forschung. Z Psychodrama und Soziometrie, 3, 109-124. Sonderheft.

Jacobsen, J.C. (2014). Systematic reviews of randomised clinical trials examining the effects of psychotherapeutic interventions versus "no intervention" for acute major depressive disorder and a randomised 
trial examining the effects of "third wave" cognitive therapy versus mentalization-based treatment for acute major depressive disorder. Dan Med J, 61(10), B4942.

Thun-Hohenstein, L. (2014). Partizipation von Kindern und Jugendlichen an einer Kinder+Jugendpsychiatrie. Pädiatrie \& Pädologie, 49, 42-47. Supplement.

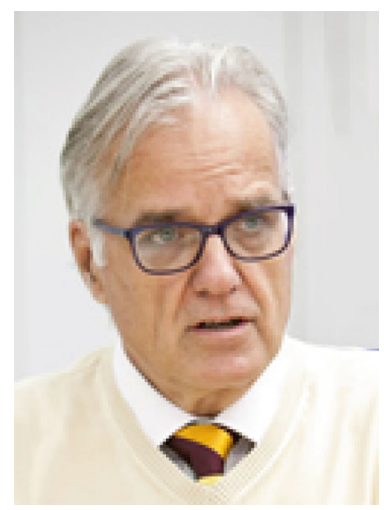

Prim. Univ.Prof. Dr. Leonhard Thun-Hohenstein ist Leiter der UK für Kinder- und Jugendpsychiatrie Salzburg und Lehrstuhlinhaber; Facharzt für Kinder- und Jugendpsychiatrie und Psychodramatherapeut

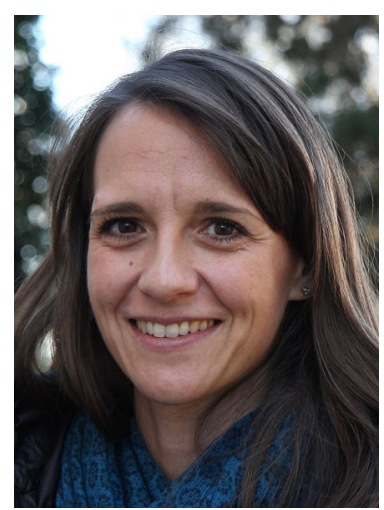

Julia Trost-Schrems ist Oberärztin an der UK für Kinder- und Jugendpsychiatrie Salzburg und leitet die Tagesklinik; Fachärztin für Kinderund Jugendpsychiatrie, Psychodramatherapeutin i.A.u.S. 